\title{
4 Chlorophyll Mutations
}

There are two important goals of mutation research viz. the enhancement of mutation frequency and alteration of mutation spectrum in a predictable manner. In the past, different approaches have been tried to achieve these goals (Gustafsson, 1963; Nilan, 1967; Swaminathan and Sharma, 1968; Chakarbarti, 1975; Maluszynski, 2001; Mehandjiev et al., 2001). The scoring of chlorophyll mutation frequency in $M_{2}$ generation is one of the most reliable measures for evaluating the mutagenic induced genetic alterations of the mutagen treatments used on the plant ideotype (Chaturvedi and Singh, 1990). Chlorophyll deficient chimers in $M_{1}$ generation and their segregation in $\mathrm{M}_{2}$ generation are often observed in a mutagenized population. Several authors have reported the occurrence of different types of chlorophyll mutations such as albina, xantha, chlorina, viridis, virescent, tigrina etc., in $\mathrm{M}_{2}$ generation following treatments with various mutagenic agents in different crop species. Some of the noteworthy examples are of Dahiya (1973) and Wani et al. (2011c) in mungbean; Venkateshwarlu et al. (1978) in pigeonpea; Venkateswarlu et al. (1988) in Catharanthus roseus; Prasad and Das (1980), Bawankar and Patil (2001) and Waghmare (2001) in grasspea; Dixit and Dubey (1986b), Reddy et al. (1993) and Paul and Singh (2002) in lentil; Arora and Kaul (1989) in Pisum sativum; Anwar and Reddy (1981), Reddi and Suneetha (1992) and Yamaguchi et al. (2006) in rice; Mohanasundaram et al. (1998), John (1999) and Girija and Dhanavel (2009) in cowpea; Singh et al. (1999) in urdbean; Prakash and Shambulingappa (1999) and Devi et al. (2002) in rice bean; Szarejko and Maluszynski (1999) in barley; Deepa and Devi (2000) in winged bean; Kumar et al., (2003) in limabean; Toker and Cagirgan (2004b), Khan et al. (2005b), Barshile et al. (2006), Bhat et al. (2012) in chickpea and Khan and Tyagi (2009b) in soybean. The frequencies of chlorophyll mutations in different crops with different mutagens have been found to be markedly different. Chemical mutagens induce higher frequency of chlorophyll mutations than radiations (Blixt, 1972; Filippetti et al., 1977; Sharma and Sharma, 1981b; Tripathi and Dubey, 1992; Vandana et al., 1994; Kharkwal, 1998b; Singh et al., 2000a; Waghmare and Mehra, 2001; Karthika and Subbalakshmi, 2006; Lal et al., 2009; Arulbalachandran and Mullainathan, 2009a). Among the chemical mutagens, EMS was reported to induce a higher frequency of chlorophyll mutations. The superiority of EMS in inducing chlorophyll mutations at a higher frequency was also suggested by Swaminathan et al. (1962), Muntzing and Bose (1969), Hussein et al. (1974), Reddi and Suneetha (1992), Shah et al. (2006), Gupta (2008), and Wani et al. (2011b). Ionizing radiations generally produce a higher proportion of albina mutations than chemical mutagens (Swaminathan et al., 1962; Ando, 1970; Gupta and Yashvir, 1975; Subramanian, 1980; Cheema and Atta, 2003; Karthika and Subbalakshmi, 2006). However, Hemavathy and Ravindran (2005) found that the occurrence of albina is less compared to the chlorina and xantha in gamma rays treated population of Vigna mungo.

(c))BY-NC-ND $($ C 2014 M. Imran Kozgar

This work is licensed under the Creative Commons Attribution-NonCommercial-NoDerivs 3.0 License. 
The use of combination treatments of physical and chemical mutagens alters the mutation frequency and spectrum (Arnason et al., 1963; Favert, 1963) and has gained a great momentum in its usage in the recent past in mutation breeding programmes. Chemical mutagens when combined with radiation are not only mutagenic themselves but also affect mutation in specific ways and are more effective in inducing chlorophyll mutations comparing to individual treatments (Sharma, 1969; Khalatkar and Bhatia, 1975 Reddy and Smith, 1981; Singh et al., 1999; Khan and Tyagi, 2009b). Similar observations of synergistic effects were made on rice treated with thermal neutrons and diethyl sulphate, DES (Rao and Ayenger, 1964) and on black gram in the combination treatments of gamma rays and EMS (Gautam et al., 1992). In some cases, the antagonistic effects have also been reported in the combined treatments of gamma rays and EMS (Arora and Kaul, 1989). However, in general, most of the mutagens given in combination exhibit synergism in legumes and cereals.

There is a strong indication that total mutations frequency and spectrum are associated with the dose of mutagen (Hussein et al., 1974; Sarma et al., 1979; Reddy and Gupta, 1989; Reddy and Revathi, 1991; Venkatachalam and Jayabalan, 1993; Singh et al., 1999; Amarnath and Prasad, 2000; Das and Kundagrami, 2000). However, this claim was repudiated by some workers (Pipie, 1972; Khan, 1990; Yamaguchi et al., 2009) who found no relationship between the dose of mutagen and the mutation spectrum. According to Gaul (1964), Vo Hung (1974), Khan and Siddiqui (1993), Kaul and Bhan (1977), Khan (1986), Reddi and Suneetha (1992), Raveendran and Jayabalan (1997), Mitra and Bhowmik (1999) and Ganapathy et al. (2008), the highest dose is not always the most effective treatment.

\subsection{Case Study 2}

Chlorophyll mutations are one of the reliable indices to assess the genetic effects of mutagenic treatments. The seeds of chickpea were harvested from the Pusa-256 and BG-1053 varieties in $M_{1}$ generations as per Case Study 1 and were sown in $M_{2}$ generations and the progenies developed were screened for the chlorophyll mutations as per methodologies explained in Appendix. The outcome results and discussion are given herewith.

\subsubsection{Results}

Six different types of chlorophyll mutants were recorded in the field in $\mathrm{M}_{2}$ generation when seedlings were 10-20 days old. The spectrum of different $\mathrm{M}_{2}$ chlorophyll mutants included: albina, chlorina, maculata, tigrina, viridis and xantha. All these chlorophyll deficient mutants were lethal except chlorine, maculata and viridis. A brief description of the isolated different chlorophyll mutants is given in Table 4.1 and Fig. 4.1. 
Table 4.1: Characteristic features of chlorophyll mutants and percentage mutated plant progenies induced by gamma rays, EMS and their combinations in two varieties of chickpea in $M_{2}$ generation.

\begin{tabular}{llll}
\hline $\begin{array}{l}\text { Isolated mutant types and } \\
\text { their characteristics }\end{array}$ & Treatment & $\begin{array}{l}\text { No. of } \\
M_{1} \text { plant } \\
\text { progenies }\end{array}$ & $\begin{array}{l}\text { No. of plant } \\
\text { progenies mutated } \\
\text { segregating plant } \\
\text { in } M_{2}\end{array}$ \\
& & & $\left(M_{p}\right)$ \\
\hline
\end{tabular}

\section{Albina}

Lethal mutation characte-

rized by entirely white leaves Control

of seedlings; seedlings

survived for 10-12 days after

germination.

$100 \mathrm{~Gy} \gamma$ rays

200 Gy $\gamma$ rays

$300 \mathrm{~Gy} \gamma$ rays

2. Chlorina

$400 \mathrm{~Gy} \gamma$ rays

Light green colour of leaves;

most of the seedlings died

$0.1 \%$ EMS

within 20 days. However, few

vigorous plants survived and

were late in maturity.

$0.2 \%$ EMS

$0.3 \%$ EMS

$0.4 \%$ EMS

3. Maculata

Seedlings showed yellow

or whitish dots on leaves;

These mutants survived till maturity and produced few seeds.

$100 \mathrm{~Gy}$ y rays $+0.1 \%$ EMS

$100 \mathrm{~Gy}$ y rays $+0.2 \%$ EMS

$200 \mathrm{~Gy}$ Y rays $+0.1 \%$ EMS

$200 \mathrm{~Gy}$ Y rays $+0.2 \%$ EMS

Var. Pusa-256

50

50

50

50

50

50

50

50

50

50

50

50

50

Var. BG-1053

\section{Tigrina}

Leaves yellow with green patches typical of the colour of the skin of Indian frog; survived for 15 days.

\section{Viridis}

Reduced plant height and viridine green colour of leaves; leaflets size reduced; plants were slow growing and had a low seed yield.

\section{Xantha}

Leaves were bright yellow in colour; seedlings survived for 10-20 days.

\section{Control}

$100 \mathrm{~Gy} \gamma$ rays

200 Gy $\gamma$ rays

300 Gy $\gamma$ rays

400 Gy $\gamma$ rays

\section{$0.1 \%$ EMS}

$0.2 \%$ EMS

$0.3 \%$ EMS

$0.4 \%$ EMS

100 Gy $\gamma$ rays $+0.1 \%$ EMS 100 Gy $\gamma$ rays $+0.2 \%$ EMS 200 Gy $\gamma$ rays $+0.1 \%$ EMS 200 Gy rays $+0.2 \%$ EMS
50

50

50

50

50

$$
50
$$

50

50

50

\section{0}

50

50

50
2.0

8.0

10.0

10.0

2.0

8.0

8.0

10.0

12.0

14.0
6.0

14.0

16.0

16.0

4.0

12.0

12.0

14.0

12.0

14.0

14.0

16.0 

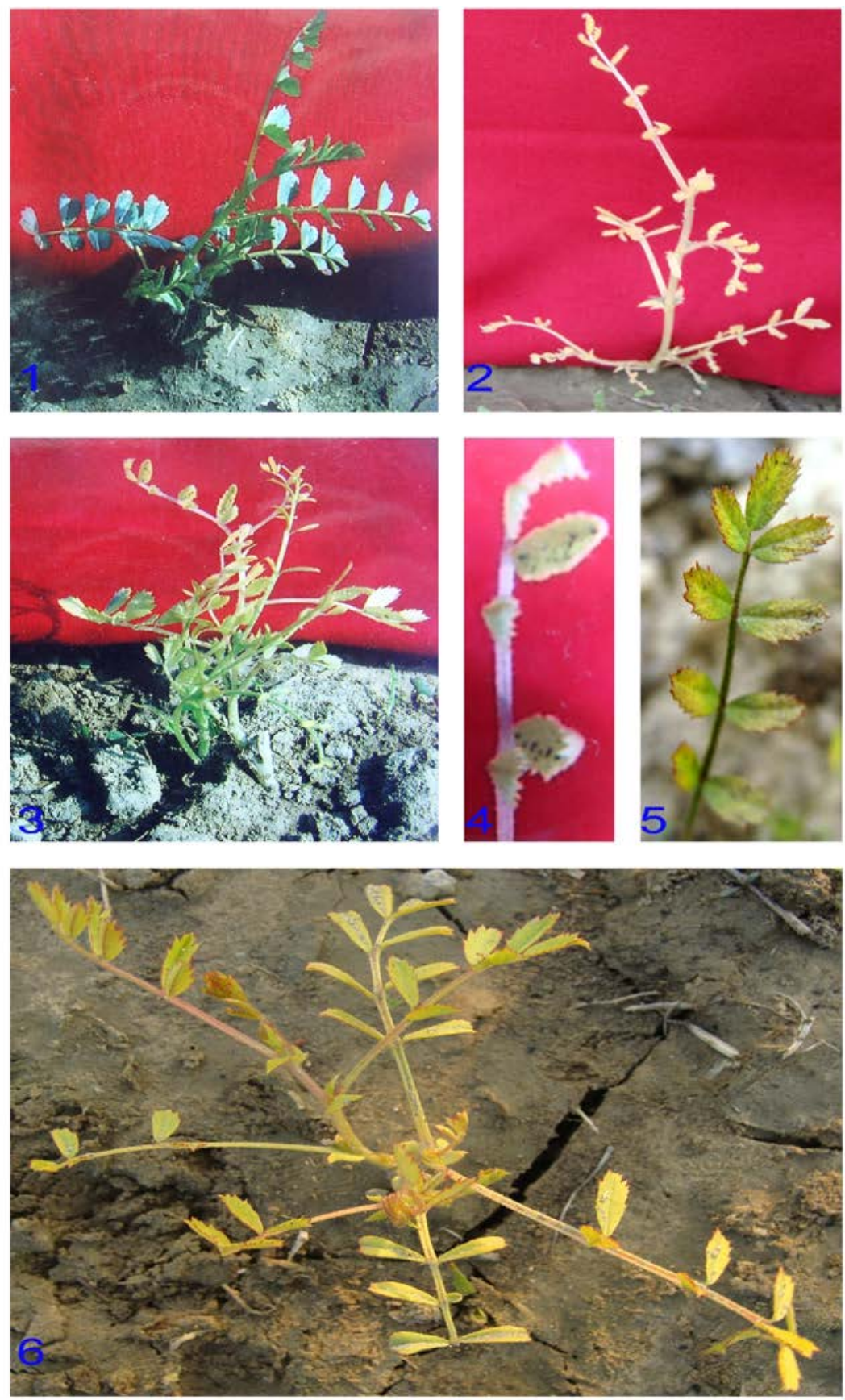

Fig. 4.1: Chlorophyll mutants: 1. Control seedling; 2. Albina mutant (white leaves of seedling); 3. Chlorina mutant (light green colour); 4. Tigrina mutant (patches of green dots on leaflets); 5. Viridis mutant (viridine green colour); 6. Xantha mutant (bright yellow colour). (Photographs by M I Kozgar) 
The chlorophyll mutations frequency was calculated on progeny basis as well as on $\mathrm{M}_{2}$ seedling basis. The trend of the mutation frequency was similar in both of the methods (progeny basis as well as on $\mathrm{M}_{2}$ seedling basis). Therefore, results are presented on $\mathrm{M}_{2}$ plant basis. A linear relationship between mutations frequency and the dose of gamma rays, EMS and their combinations was observed in both varieties of chickpea up to $300 \mathrm{~Gy}$ in gamma rays treatments, up to $0.3 \%$ in EMS treatments and up to 200 Gy gamma rays $+0.1 \%$ EMS in combination treatments. Among the mutagens used, the combined gamma rays + EMS treatments gave a higher mutation frequency in comparison to individualistic treatments of gamma rays and EMS. However, the EMS treatments were found to be more effective than gamma rays treatments. Variety BG-1053 responded with the greater mutation frequency as compared with the variety Pusa-256. In both varieties the frequency (1.06\%) of albino mutants was the highest, followed by those of chlorina, xantha, maculata, viridis and tigrina. The ratio of albino/chlorine was found to be 1.51, 0.83 and 1.24 for gamma rays, EMS and gamma rays + EMS, respectively (Fig. 4.2).
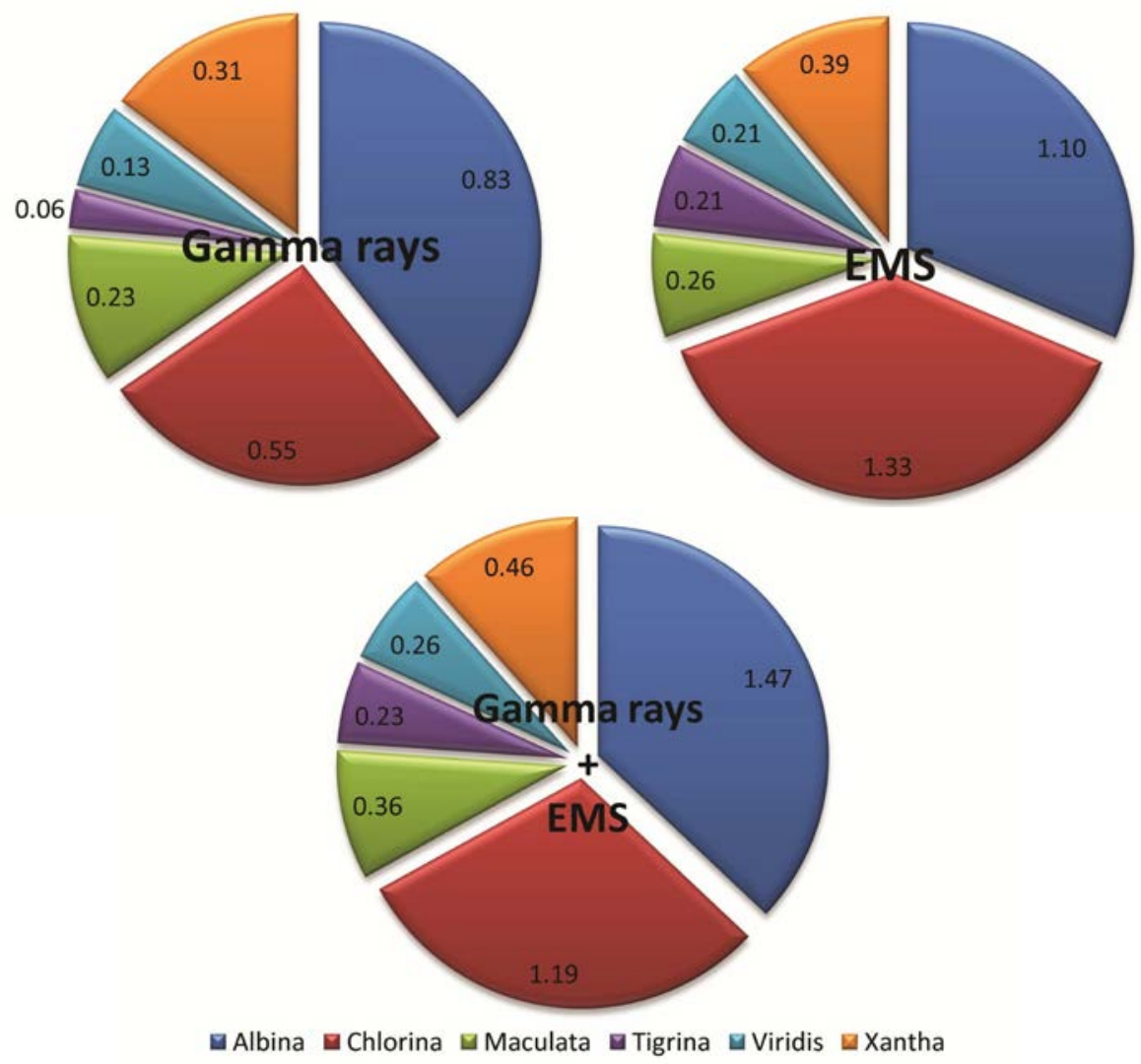

Fig. 4.2: Comparative frequency (\%) and spectrum of chlorophyll mutations in chickpea based on pooled values of two varieties of chickpea (Pusa-256 and BG-1053). 
The combined treatments did not show any additive effect in producing chlorophyll mutations. The results obtained in the present study showed negative synergism in both varieties of chickpea.

\subsubsection{Discussion}

The chlorophyll mutation frequency is useful in assessing the potency of a mutagen, genetic effects of mutagen and estimation of mutational events mainly because of ease of identification. From a breeder's point of view, the frequency of chlorophyll mutants expressed as per cent of $\mathrm{M}_{2}$ population seems to be more realistic and helpful. Therefore, results were explained on $\mathrm{M}_{2}$ plant basis. In the present study, a critical comparison of the chlorophyll mutations indicates that the mutation rate, in general, increased with an increase in dose up to a certain dose level beyond which it decreased. This trend was observed in both the varieties in treatments with gamma rays, EMS and their combination. Higher frequencies of chlorophyll mutations with medium or lower doses of mutagens were reported by Srivastava et al. (1973), Nadarajan et al. (1982), Toker and Cagirgan (2004a), Yamaguchi et al. (2009) and Pawar et al. (2010) in different crops including chickpea. The decrease in chlorophyll mutation frequency as observed at the highest doses of mutagens may be attributed to saturation in the mutational events, which may result in the elimination of the mutant cells during growth. Contrary to the results obtained, the works of Subramanian (1980), Grover and Virk (1984), Khan (1990), Khan and Siddiqui (1992), Reddy et al. (1993), Kharkwal (1998b), John (1999), Das and Kundagrami (2000), Barshile et al. (2006), Pavadai et al. (2009) and Kumar et al. (2009a) reported dose dependent increase in the chlorophyll mutations frequency with different mutagenic treatments. Occurrence of chlorophyll mutants in large number of crops have been attributed to different causes such as impaired chlorophyll biosynthesis, further degradation of chlorophyll and bleaching due to deficiency of carotenoids (Bevins et al., 1992) and may be related to their preferential action on chlorophyll development genes (Reddy and Anndurai, 1991). Recovery of a higher number of albina from combination treatments of gamma rays + EMS is contrary to the contention of Arora and Kaul (1989) that gamma rays alone or in combination with EMS induced more chlorina mutants. Athwal et al. (1970) in chickpea and/or as well as Karthika and Subbalakshmi (2006) in soybean reported that albina constituted the largest single category of mutants in gamma rays treated population. In the present study, EMS produced more albino types than gamma rays. The frequency of chlorophyll mutations induced by gamma rays was less than that of EMS and combination treatments. These results are in contrary to the findings of Arora and Kaul (1989) who observed, in Pisum sativum, that gamma rays treatment was the most potent in inducing the highest chlorophyll mutations frequency. EMS is supposed to be specific to certain chromosomal regions (Goud, 1967; Khan and Siddiqui, 1993; Uchida et al., 2011) containing genes for chlorophyll development and 
has been reported to induce high frequency of chlorophyll mutations (Swaminathan et al., 1962; Girija and Dhanavel, 2009; Pawar et al., 2010; Bhat et al. 2012).

The combined treatments of gamma rays + EMS showed a considerable degree of synergism in increasing the frequency of chlorophyll mutations. Synergistic effects of physical and chemical mutagens have been reported when they are used in combination in various crops (Khalatkar and Bhatia, 1975 in barley; Agarkova and Yakovlev, 1977 in Phaseolus vulgaris; Ignacimuthu and Babu, 1988 and Singh, 2007a in Vigna spp.; Venkateswarlu et al., 1988 in Catharanthus roseus; Reddy, 1992b in triticale). A probable reason for synergism is that the mutagen first applied may expose accessible the protected mutable sites to the second mutagen, and secondly, the repair enzymes may be rendered non-functional by the second mutagen, thereby promoting the fixation of already induced pre-mutational changes (Sharma, 1970; Makeen et al., 2010). In the present study, negative synergism (less than additive) was observed in combination treatments of gamma rays + EMS (unpublished data). Less than additive effect may result from two mutagens competing for the same site (Aastveit, 1966). The negative synergism between hydrazine (HZ) and hydroxylamine (HA) for chlorophyll mutation frequency in $\mathrm{M}_{2}$ may be explained by assuming that at least some of the mutational events produced by the first of applied mutagens might be getting reversed by the second mutagen (Reddy et al., 1973).

Discrepancies regarding the mutation frequency for even similar treatments in the two varieties of chickpea suggested a possible influence of genome on mutagenic potency. Inter-varietal differences with regards to the mutation frequency as observed in the present study confirm the findings of Singh et al. (1998) in rice, Ahmed (1999) in cowpea and Kumar et al. (2003) in limabean. Genetic differences even of a single gene induce significant changes in mutagen sensitivity, which influences not only the rate but also the spectrum of recoverable mutations (Bhan and Kaul, 1976; Paul and Singh, 2002). 